\title{
Michael D. White \& Aili Malm (2020). Cops, Cameras, and Crisis: The Potential and the Perils of Police Body-Worn Cameras. New York: New York University Press
}

\author{
Katherine Schofield \\ Monash University, Australia
}

\section{ISBN: 9781479865864}

As technology becomes progressively more entwined with society and pervades almost every aspect of our personal lives, its uptake in other areas, such as the criminal justice system, is also becoming increasingly ubiquitous. There is a need for criminologists to examine the implications of this technological creep, as the pervasiveness of technology and the normalisation of surveillance by those in authority seem to be accepted as the trade-offs for the convenience that such technology imparts. This is the context in which Cops, Cameras, and Crisis: The Potential and the Perils of Police Body-Worn Cameras is published; a book by Michael D. White and Aili Malm that provides a comprehensive review of the existing research into bodyworn cameras (BWCs) in policing. The book consists of five chapters interspersed with short vignettes that highlight the perspectives of those working in the criminal justice field with firsthand experience of BWCs. The content is supported by tables and figures that clearly illustrate the research findings.

Cops, Cameras, and Crisis begins with an overview of the use of cameras in law enforcement and the circumstances in which BWCs, specifically, have become progressively used. A watershed moment was the killing of Eric Garner and Michael Brown in 2014 by police, which ignited the Ferguson protests in the United States (US) and the Black Lives Matter movement. The timely nature of the topic explored in Cops, Cameras, and Crisis is striking given the powerful resurgence of the Black Lives Matter campaign in 2020, just one month after the book's release, following George Floyd's death at the hands of police. The movement has since reverberated around the world, with adaptations to local contexts; similarly, the response by the Obama government in the wake of Ferguson has been taken up by jurisdictions outside the US, with BWCs becoming increasingly common in policing.

After recounting the history of the widespread deployment of BWCs in policing, Chapter One turns to the evidence base. White and Malm introduce the concept of evidence-based policing as 'a body of knowledge that applies rigorous research methodologies to the study of innovation in policing " - the lens through which they explore police BWCs. Delving into the

\footnotetext{
${ }^{1}$ White and Malm, Cops, Cameras, and Crisis, 2.
} 
claims of advocates and critics, Cops, Cameras, and Crisis seeks to provide 'an exhaustive examination of the research base', ${ }^{2}$ and indeed, a comprehensive digest of the existing evidence for police use of BWCs is outlined.

The reported benefits of police BWCs are examined in Chapter Two, particularly the 'pertinent questions of police transparency, accountability, and legitimacy'. ${ }^{3}$ Evaluation of the evidence shows that police BWCs do increase these three elements, or at least the perception of them. However, there are crucial failings in the operationalisation of these concepts in existing research. Easier to operationalise is police use of force and complaints against police, and therefore these issues have 'eclipsed other areas of BWC research'. ${ }^{4}$ The next section sees White and Malm explore the claim that BWCs lead to 'less use of force by police, fewer citizen complaints, less resistance by citizens, and ... fewer injuries and fatalities' ${ }^{5}$ The potential evidentiary value of BWCs is positioned as something that 'can help sell a BWC program to reluctant officers and unions' ${ }^{6}$ However, White and Malm admit that 'rigorous evidence supporting these claims is scant' ${ }^{7}$ and that 'research in this area is still pretty thin'. ${ }^{8}$ While touching on the benefits of the technology for citizens, more dominant is the focus on how this technology benefits police practice. Although these can overlap in some situations, this chapter is missing a critical exploration of how BWCs might benefit police at the expense of citizen rights.

Despite the potential benefits of police BWCs, 'if implemented poorly, [they] can actually make matters worse'. ${ }^{9}$ In Chapter Three, the technology's limitations are examined. In reviewing the costs of BWCs, the dilemma between rights to privacy and increased transparency is raised. White and Malm review the evidence for key policy issues, and it becomes obvious here that Cops, Cameras, and Crisis is predominantly aimed at a readership of police leaders and policymakers. For example, it is stated that 'a well-formed, well-researched policy can help protect both the officer and department from critique and litigation', ${ }^{10}$ which is a curious way to frame the issue. Good policy should not be about protecting those in positions of authority from criticism and/or legal action, but about serving and protecting citizens. Although it is good that camera footage could exonerate any police officers accused of acts that they have not committed, I find it perplexing that criminologists would not position the protection of citizens as a priority for police policy.

BWCs are 'a form of surveillance that allows for internal (by supervisors) and external (public) scrutiny of officer behavior,' 11 and this may lead to depolicing, in which officers disengage from active police work. Alternatively, they might 'increase their formal activity (stops, citations, arrests, etc.) rather than handling encounters informally (e.g., warnings)', ${ }^{12}$ knowing that supervisors could review BWC footage. Evidence for these two competing concerns is once again mixed. It would have been good to see White and Malm critically engage with the concept of depolicing and the related, so-called 'Ferguson effect', which have both featured heavily in US political rhetoric in recent years, despite academic debate on their validity. ${ }^{13}$ The discussion about engaging with external stakeholders when developing police BWC programs is useful, although many of the specifics are less applicable to those outside the US due to differences in institutional structures and operation.

Cops, Cameras, and Crisis successfully outlines the key practical benefits and limitations of police BWCs identified in research thus far, and the readability of the book is enhanced by its logical progression. This is with the exception of Chapter Four, which, inexplicably at this late stage in the book, introduces theory to explain the adoption of police BWCs and whether their proliferation is likely to continue. White and Malm continue to look to the future in Chapter Five, anticipating the 'most serious emerging threats to successful BWC programs' ${ }^{14}$ as activation compliance, managing the public's expectations, and technological advances that 'push BWCs into new areas'. ${ }^{15}$ The most pressing concern is the fact that 'most police departments

\footnotetext{
${ }^{2}$ White and Malm, Cops, Cameras, and Crisis, 12.

${ }^{3}$ White and Malm, Cops, Cameras, and Crisis, 17.

${ }^{4}$ White and Malm, Cops, Cameras, and Crisis, 27.

${ }^{5}$ White and Malm, Cops, Cameras, and Crisis, 28.

${ }^{6}$ White and Malm, Cops, Cameras, and Crisis, 43.

${ }^{7}$ White and Malm, Cops, Cameras, and Crisis, 43.

${ }^{8}$ White and Malm, Cops, Cameras, and Crisis, 45.

${ }^{9}$ White and Malm, Cops, Cameras, and Crisis, 50.

${ }^{10}$ White and Malm, Cops, Cameras, and Crisis, 59.

${ }^{11}$ White and Malm, Cops, Cameras, and Crisis, 70.

${ }^{12}$ White and Malm, Cops, Cameras, and Crisis, 70.

13 Tiwari, "Protests, Policing, and Crime."

${ }^{14}$ White and Malm, Cops, Cameras, and Crisis, 103.

${ }^{15}$ White and Malm, Cops, Cameras, and Crisis, 103.
} 
have not fully considered the cybersecurity issues surrounding their BWC data-storage systems ${ }^{16}$ and White and Malm provide a good overview of the terrifying repercussions of lax security. Nevertheless, no detailed solutions are provided, aside from mentioning best-practice standards developed by Swire and Woo (2018) for the Internet of Things that can be extended to BWC footage. ${ }^{17}$ This criticism also applies to the discussion about how BWC data might be used to collect and analyse biometric metadata, particularly facial recognition. White and Malm identify the 'lack of legal restraint on the scope and potential applications of bulk biometric data collection initiatives', ${ }^{18}$ stating Hu's (2018) argument that BWC data could 'serve as the foundation for systematized biometric data analysis that would provide law enforcement and the intelligence community with mass, suspicionless surveillance of ... citizens'. ${ }^{19}$ It is a confronting thought, but, once again, suggestions are not given on how to prevent this from occurring. Despite acknowledging the concerns presented in this chapter as legitimate, no rationale is offered as to why the benefits of police BWCs outweigh the negative potential of implementing the programs White and Malm advocate.

Cops, Cameras, and Crisis states that 'BWCs will become a permanent part of the policing landscape', ${ }^{20}$ and it is from this assumption that White and Malm work, outlining how to implement police BWC programs grounded in evidence. Advice is targeted at police leaders, policymakers and practitioners, recognising that most, if not all, police departments will eventually adopt BWCs into their arsenal. Although White and Malm are critical of the quality of research, they do not engage critically with the ethical questions that naturally arise from authority's use of surveillance technology. When they come close to doing so, it is often in a way that positions police concerns over those of citizens. It is remiss to neglect these issues, given the effect that misuse can have on people's ability to acquire justice, particularly those from marginalised communities. Even in a book aimed at police leadership, it is surprising to see criminologists pay such little attention to the inherent power imbalance between law enforcement and those they police. While Cops, Cameras, and Crisis examines the evidence to evaluate whether BWCs can be used to improve policing, it remains necessary to question whether BWCs should be used in policing.

The strength of Cops, Cameras, and Crisis lies in its detailed review of the existing evidence for police BWCs, and this will be useful to all readers with an interest in the topic. White and Malm are thorough in their research, clearly presenting what is known and what requires further study, as well as recommendations for policy development. Some elements of this advice do not apply beyond the US context, where police are funded on a municipal rather than just a state or federal level (as occurs in Australia). Nevertheless, the more general suggestions provided in Cops, Cameras, and Crisis have broader applicability and are of benefit to those in the practitioner space. Overall, White and Malm succeed in achieving their key aim of providing a comprehensive examination of the research base to facilitate evidence-based practice regarding BWCs in policing. There remains a need for future work to consider the broader implications of police BWCs through a more critical lens, exploring how such technology can be misused by those in positions of authority and the ethical issues that arise from an increasingly surveilled society.

\section{Bibliography}

Hu, Margaret. "Bulk Biometric Metadata Collection.” North Carolina Law Review 96, no. 5 (2018): 1425-1476. Quoted in White, Michael D., and Aili Malm. Cops, Cameras, and Crisis: The Potential and the Perils of Police Body-Worn Cameras. New York: New York University Press, 2020.

Swire, Peter, and Jesse Woo. "Privacy and Cybersecurity Lessons at the Intersection of the Internet of Things and Police Body-Worn Cameras." North Carolina Law Review 96, no 5 (2018): 1475-1523.

Tiwari, Alisa. "Protests, Policing, and Crime: An Analysis of Evidence Regarding the Ferguson Effect." Journal of Public and International Affairs 27, no 1 (2016): 115-131.

White, Michael D., and Aili Malm. Cops, Cameras, and Crisis: The Potential and the Perils of Police Body-Worn Cameras. New York: New York University Press, 2020.

\footnotetext{
${ }^{16}$ White and Malm, Cops, Cameras, and Crisis, 114.

${ }^{17}$ Swire and Woo, "Privacy and Cybersecurity Lessons."

${ }^{18} \mathrm{Hu}$,"Bulk Biometric Metadata Collection," 1443.

${ }^{19}$ White and Malm, Cops, Cameras, and Crisis, 115.

${ }^{20}$ White and Malm, Cops, Cameras, and Crisis, 16.
} 